intensity of blue light at Washington on the average day seems to indicate that there is a substantial amount of scattering by small solid or liquid particles.

The figures for Mount Wilson give us confidence in the trustworthiness of Mr. Abbot's determination of the solar constant, because it is clear that the total effect of the atmosphere can be eliminated with greater certainty if it is mainly due to the permanent constituents of the atmosphere, and not to matter which is variable in amount. As the top of Mount Wilson is less than 1800 metres above sea-level, we may conclude that at high elevation the blue colour of the sky is completely accounted for by molecular scattering.

That the scattering sets a limit to the transparency of gases must be kept in mind in discussing problems of solar and stellar physics. We may feel confident, for example, that what is called the reversing layer can only have a small thickness, for otherwise we should not be able to observe so far into the ultra-violet as we do.

The scattering may profoundly modify the character of the spectrum, as I have explained in a paper on " Radia tion through a Foggy Atmosphere" (Astrophysical Journal, vol. xxi., p. 1), in which it is shown how increased thickness, without change of temperature, may convert an absorption line into a bright line. It seems to me probable that the bright and dark flocculi shown in spectroheliograph pictures may find their explanation chiefly in variation of thickness in the absorbing layer, the bright patches being due to increased thickness.

The transparency of interstellar space has recently received a much needed critical discussion at the hands of astronomers, and Prof. Turner has applied the law of scattering to explain certain discrepancies between visual and photographic magnitudes. The value he gives for the opacity allows us to calculate the average density of the matter which is diffused through space on the supposition that it is gaseous. If the value of $(\mu-1) / D$ (where $\mu$ is the refractive index and $\mathrm{D}$ the density) be taken to be approximately equal to that of air, I find that the number of molecules per cubic centimetre in space would have to be of the order of a million, and the mean free path of the order of 3000 kilometres.

Although not directly connected with the subject which forms the main part of this communication, I would like to point out that the same analysis which gives the coefficient of extinction in terms of the retardation of phase at the source of the scattering also gives a resultant force acting on the molecule in the direction in which the light is passing. When summed up for all the molecules this force is found to be identical with what is generally called the "pressure of light," for if E represents the energy density, the force acting per unit volume on the scattering molecules is found to be $k \mathrm{E}$, where $k$ is the coefficient of extinction.

There is a widespread impression that light pressure only acts on particles the linear dimensions of which include several wave-lengths of light, but this is not correct. The determining factor is the extinction of light, whether by scattering or by absorption, as indeed appears if we take the view adopted in Prof. Poynting's work on the subject that a propagation of momentum accompanies the transmission of light. The momentum is destroyed equally whether the molecules act as scattering or as absorbing centres. The extinction by scattering near the surface of stellar bodies does not, however, appear to be sufficient to cause any measurable effects comparable with their gravitation.

ARthur Schuster.

\section{The Fixation of Nitrogen by Soil Bacteria.}

MAY I be allowed through the columns of NATURE to ask Prof. W. B. Bottomley a few questions with regard to his paper on "Some Effects of Nitrogen-fixing Bacteria on the Growth of Non-leguminous Plants" (Proc. Roy. Soc., B, Ixxxi., I909, 287), abstracted in Nature of May I3 (vol. Ixxx., p. 327), as I had not the opportunity of being present when the paper was read?

Prof. Bottomiley bases his conclusions on experiments to show that Pseudomonas, the bacteriurm associated with the ledumincus plants, will fix mere nitrogen in an artificial No. 2073 , vOL. 81$]$ culture, when Azotobacter is also present, than when alone. He quotes the following results:-

Control ... $\quad \ldots \quad \ldots \quad 0.48 \mathrm{mgm}$. N. per 100 c.c. culture solution Pseudomonas alone 0.91 , , , Pseudomonas and

Azotobacter $\quad . . . \quad$ I'24

, " , ,

These differences would almost seem to be within the range of experimental error, but in any case, does not the demonstration require a further statement of how much nitrogen Azotobacter alone would fix? Other observers are accustomed to get fixations by Azotobacter alone of from 5 to $20 \mathrm{mgm}$. of nitrogen per 100 c.c. of such a culture solution, the maximum being about ro $\mathrm{mgm}$. of nitrogen fixed per gram of mannite. The only conclusion that could be drawn from Prof. Bottomley's figures would be that Pseudomonas injuriously affects the power of Azotobacter to fix nitrogen, supposing that a reasonably active culture of the latter had been used.

Turning to the field experiments, in which Prof. Bottomley claims to get an increase of crop by adding cultures of Pseudomonas and Azotobacter to soil which presumably already contains both organisms; data for estimating the probable experimental error are again lacking. From the Rothamsted experiments, where we may assume the conditions are more than usually favourable to exactitude, the mean error of a pair of similarly treated plots in a single year is about \pm ro per cent., which would more than cover the differences observed by Prof. Bottomley's experiment with oats.

In another experiment with barley, Prof. Bottomley obtained a higher percentage of nitrogen in the corn from the treated strip than in the corn from the rest of the field, $\mathrm{I} .76$ against $\mathrm{I} .55$ per cent. (I presume that " milligrammes of nitrogen per cent." is a clerical error). In view of the comparatively small changes in the composition of the grain of a cereal which are usually effected by large variations in the supply of nutrient, I should like to know from Prof. Bottomley if duplicate samples were taken from different parts of the untreated section of the field, and what range of variation they showed in their nitrogen content.

Coming to the next experiment, it is difficult to judge how far a bulbous plant like Galtonia candicans is suitable for experiments on nutrition, but it is rather necessary to know what relation the weight of the bulbs planted bore to those harvested. Prof. Bottomley only says that 250 bulbs " of equal size" were planted in each bed. Can he let us have the weights in each case? Moreover, he tells us that the treated bed was twice watered with the culture solution, the control bed being given pure water at the same time; was the same amount of water given to each, and how much of the culture solution was applied, for it contained monopotassium phosphate, sodium chloride, \&c., which may well have been a considerable factor in any beneficial effect experienced?

Prof. Bottomley will perhaps forgive me if these questions may seem somewhat critical of his conclusions, but any communication appearing in the Proceedings of the Royal Society must be taken into account, and one therefore wishes to have the data necessary for determining the weight to be attached to the results. A. D. HALL.

The Rothamsted Experimental Station, July 12, 1909.

Occasional Unexplained Ringing of House-bells.

AN observation sent me by Mr. Alexander Sinclair, of Swansea, to the effect that during a thunderstorm drops of water leaking through the ceiling " assumed a pear shape and jumped 9 inches almost horizontally to the curtain rings above the window," suggests that house-bells of the ordinary non-electric type may occasionally be rung by this means. I picture the process as follows :-The belf wires collect atmospheric electricity, by induction or otherwise, which the walls are insufficiently conducting to carry off freely; consequently the bells get charged, are attracted to a neighbouring wall or pipe, and released suddenly by a spark. This little lateral jerk rings the bell.

I put the simple suggestion on record because I sometimes hear of an inclination to attribute the phenomenon to less familiar causes.
Oriver lodge. 